\title{
Correction to: Study on the influence of different menu sizes on the operation performance of touch screen
}

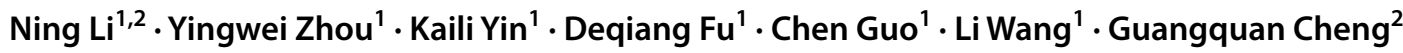

๑) Springer-Verlag GmbH Germany, part of Springer Nature 2019

Correction to: Evolutionary Intelligence

https://doi.org/10.1007/s12065-019-00316-4

The article, Study on the influence of different menu sizes on the operation performance of touch screen, written by Ning Li, Yingwei Zhou, Kaili Yin, Deqiang Fu, Chen Guo, Li Wang, Guangquan Cheng, was originally published electronically on the publisher's internet portal (currently SpringerLink) on 27 November 2019 with open access. With the author(s)' decision to step back from Open Choice, the copyright of the article changed on 26 December 2019 to $($ ) Springer-Verlag GmbH Germany, part of Springer Nature 2019, and the article is forthwith distributed under the terms of copyright. The original article has been corrected.

The original article can be found online at https://doi.org/10.1007/ s12065-019-00316-4.

Guangquan Cheng

chengguangquannudt@163.com

Ning Li

lining199008@163.com

Yingwei Zhou

zhouyingweibh@163.com

Kaili Yin

yinkaili1012@163.com

Deqiang Fu

fudq_accepted@163.com

Chen Guo

373234068@qq.com

Li Wang

532470103@qq.com

1 Marine Human Factors Engineering Laboratory, China Institute of Marine Technology and Economy, Beijing, China

2 College of Systems Engineering, National University of Defense Technology, Changsha, China
Publisher's Note Springer Nature remains neutral with regard to jurisdictional claims in published maps and institutional affiliations. 